\title{
Acompañamiento especializado con jóvenes en exclusión en Debabarrena: una experiencia de investigación-acción
}

\author{
Aitor Ávila Ruiz \\ Programa de Intervención Socioeducativa en Medio Abierto de la \\ comarca de Debabarrena, Kalexka Elkartea \\ <aitoravila@kalexka.com>
}

\begin{abstract}
Artikulu honek 2012 eta 2014 urteen artean garatutako lan-prozesuaren emaitza islatzen du. Aipatu prozesua Kalexka Elkarteko koordinatzaile taldeak garatu du xedetzat izanik izaera soziohezitzailea duen eskuartze-programa abian jartzea sozialki baztertuak izateko arriskuan dauden gazteen artean. Beraien beharren inguruko diagnostiko edo ikerketa batean oinarritzen da lan hau, eta lagungarri eta baliagarriak dira bertatik ateratako ondorioak eskuartzea gidatu eta bideratzeko; arestian aipatu bezala, jomuga litzateke gazteen gabeziei erantzun egokia emango dien zerbitzua ezartzea. Ikerketa honen harira, izaera sozio-hezitzailea duen eskuartzea jarri zen martxan, gaur egun ebaluatzen ari dira proiektu pilotua, berau hobetu eta arrisku egoeran dauden gazteen inklusioa bultzatuko duen zerbitzua egonkortu eta finkatzeko. Horrez gain, eta bukatzeko, hainbat ideia edo elementu erantsi eta partekatu nahi ditugu beste testuinguru batzuetan eskuartzeko beharraren inguruko hausnarketarako; halaber, egiturazko elementuak eskaintzea, izan ere, bazterturik dauden gazteen inklusioa posible egin dezaketen bide-orriak definitzeko adierazgarri izan daitezke.
\end{abstract}

\section{GAKO-HITZAK:}

Esku hartze sozio-hezitzailea, gazteak, inklusio soziala, kale-heziketa, eredu proaktiboa, ikuspegi komunitarioa.
Este artículo presenta el resultado del equipo de coordinación técnica de Kalexka Elkartea durante los años 2012-2014 para la puesta en marcha de un programa de intervención socioeducativa con jóvenes en situación de exclusión. Parte de una investigación o diagnóstico de necesidades enfocado a obtener orientaciones útiles de cara a la implantación de servicios que den respuestas adecuadas a los déficits de los/as jóvenes. Este estudio derivó en la puesta en marcha de un proyecto piloto de intervención socioeducativa, que en la actualidad se está evaluando con el objetivo de optimizar y afianzar un servicio estable que posibilite procesos inclusivos de calidad con jóvenes en situación de vulnerabilidad. Igualmente, este artículo pretende reflexionar sobre la necesidad de intervenir en otros contextos, así como sobre la dotación de elementos estructurales que hagan posible itinerarios inclusivos con jóvenes en situación de exclusión.

\section{Palabras Clave:}

Intervención socioeducativa, jóvenes, inclusión social, educación en medio abierto, modelo proactivo, enfoque comunitario. 


\section{Introducción}

Este artículo describe el proceso desarrollado por Kalexka Elkartea («http://www.kalexka.com») para la puesta en marcha de un servicio de intervención socioeducativa con jóvenes. Para llevar a término dicho proceso, se ha desarrollado una metodología basada en la investigación-acción, que, bajo nuestro enfoque, es imprescindible para ajustar la implantación de programas o servicios que ofrezcan respuestas a las necesidades de los/as destinatarios/as (y no tanto a la demanda o el síntoma). Con este ejercicio, se reconoce a las personas como protagonistas de la adquisición y fortalecimiento de sus recursos y capacidades, que les permite soslayar los déficits y culminar con éxito procesos inclusivos, todo lo cual revierte en la mejora de su calidad de vida, y por extensión, en la potenciación de la propia comunidad.

En la dilatada trayectoria de la entidad, habitualmente ha sido la Administración la que nos ha encargado desarrollar las intervenciones, pero este proyecto nació de la inquietud de la propia entidad y de sus educadores/as sociales, con larga trayectoria profesional, que han visto cómo procesos 'mimados' durante la infancia y adolescencia se bloqueaban en su desarrollo por la falta de servicios de acompañamiento en la juventud. Las fases en las que se ha enmarcado este proceso de investigación-acción han sido las siguientes:

- Diagnóstico: para empezar, se planteó contrastar la visión de diferentes profesionales de la educación social en medio abierto sobre los déficits de los/as jóvenes de 18 a 25 años, mediante la realización de un estudio (Ávila Ruiz, 2013) ${ }^{1}$ que clarificara las necesidades reales de este grupo etario en la Comunidad Autónoma del País Vasco y orientara sobre formatos de intervención socioeducativa que podrían subsanarlas (octubre 2012 - junio 2013).

- Intervención: recogiendo una de las orientaciones derivadas del estudio, se propuso desarrollar un programa piloto de intervención socioeducativa en medio abierto (educación de calle) en la comarca de Debabarrena (Gipuzkoa)² para cerciorar la validez de estas prácticas en la mejora de las condiciones de vida de los/as jóvenes (septiembre-marzo 2014).

- Evaluación: finalmente evaluamos el programa piloto para definir orientaciones de cara a optimizar el programa y el avance en prácticas socioeducativas que fortalecieran los procesos socioeducativos establecidos con los/as jóvenes, facilitando su inclusión y participación en la comunidad (marzo 2014). Vasco.

${ }^{1}$ Financiado por el Departamento de Política Social del Gobierno

${ }^{2}$ Financiado por el Área de Inclusión Social de la Diputación Foral de Gipuzkoa y el Departamento de Servicios Sociales del Ayuntamiento de Elgoibar (Gipuzkoa).

\section{Fase de diagnóstico: estudio de necesidades de jóvenes no emancipados de 18 a 25 años de la CAPV}

\section{1. ¿Por qué un estudio de necesidades en este colectivo concreto?}

La idea de realizar un estudio de necesidades de la juventud no emancipada de 18 a 25 años de la CAPV surge de la propia experiencia en el desarrollo de intervenciones por parte de los/as profesionales que componen la entidad. Kalexka es una organización sin ánimo de lucro que lleva gestionando programas de intervención socioeducativa desde 2001. En la trayectoria de la entidad, el grueso de las intervenciones desarrolladas ha sido con infancia, adolescencia y familia, especialmente en programas de intervención socioeducativa en medio abierto (educación de calle).

En la intervención socioeducativa en el medio abierto (la calle, recursos, servicios), aun habiendo cerrado las intervenciones con adolescentes, el contacto con ellos/as es común (compartimos el mismo territorio), por lo que hacemos un seguimiento de sus itinerarios en el tiempo, así como del desarrollo de sus procesos de emancipación (como resultado, muchos/as jóvenes nos transmiten cuáles son sus demandas y necesidades). El desarrollo de este seguimiento de itinerarios (sin ningún planteamiento interventivo, simplemente basado en la presencia en los espacios cotidianos de desarrollo de las personas) nos permitió ver ciertos déficits en la respuesta a este colectivo $y$, por lo tanto, plantearnos la elaboración de un estudio sobre jóvenes de 18 a 25 años de la CAPV.

La experiencia en programas de educación de calle nos ha mostrado cómo procesos impecables de chavales/as con apoyos socioeducativos se han desorientado tras pasar a la mayoría de edad, eliminarse drásticamente los acompañamientos y, por lo tanto, depositar la responsabilidad de su transición en el contexto familiar (ya que algunos/as no tienen una red fuerte de socialización y se encuentran excluidos del sistema escolar). Todo ello, en muchos casos, invisibiliza la situación de vulnerabilidad en la que se encuentran (por lo general, no acuden a recursos de acompañamiento, como servicios sociales de base, juventud o empleo), demostrándonos que ni la mayoría de edad corresponde a la edad vital ni las dificultades desaparecen al cumplir los 18 años. Esta idea viene reforzada por procesos de acompañamiento educativo ajustados a sus necesidades en la etapa infantil y adolescente (a cargo de profesionales como educadores/as sociales, trabajadores/ as sociales o profesores/as) que, con la mayoría de edad, se cortan drásticamente y dan lugar a situaciones de riesgo.

Estas reflexiones, junto con el contraste de datos estadísticos tales como el paro juvenil o el nivel de 'fracaso escolar', nos hicieron preguntarnos: ¿qué pasa con la promoción juvenil?, ¿se está trabajando en ello?; y por otro lado, ¿es que los/as jóvenes en exclusión no deben ser protegidos como colectivo 
con dificultades de inclusión? Así nació la idea de realizar un estudio de necesidades de la población joven de la CAPV (con especial atención a aquellos/as que se encontraban en una situación de mayor vulnerabilidad), entendiendo que, como entidad dedicada a la intervención socioeducativa, también nos correspondía cerciorarnos de las necesidades reales de la comunidad y transferirlas a la administración pública para que les diera respuesta ${ }^{3}$.

\subsection{Descripción general del estudio}

La entidad no pretendía en ningún momento realizar un estudio sociológico, sino un diagnóstico previo de las necesidades socioeducativas de la juventud del País Vasco en general, y en particular de aquellos/as jóvenes que se encontraban en una situación de dificultad, con el objetivo de detectar sus necesidades y dar una serie de orientaciones enfocadas al establecimiento de futuros procesos de intervención socioeducativa que pudieran adaptarse a la especificidad de cada uno de los municipios o barrios donde se llevaran a cabo. Con ello queremos decir que, desde un principio, el análisis no ha buscado ofrecer una foto de la realidad joven, sino ahondar en aquellas dificultades que tiene este grupo etario en el tránsito a la vida adulta y la emancipación, y poder así definir criterios técnicos que orientaran posibles intervenciones de carácter socioeducativo que acompañaran dichos procesos.

\subsection{Objetivos}

El objetivo principal del estudio ha sido realizar un análisis en torno a la prevención-intervención, a partir de las necesidades sociales y riesgos de exclusión de los/as jóvenes mayores de edad no emancipados de 18 a 25 años de la CAPV. Para lograrlo, se decidió:

- Recoger información sobre estudios de necesidades, normativa, documentación sobre el tema, así como recursos, servicios y agentes existentes en el conjunto de sistemas y políticas en relación al acompañamiento del colectivo juvenil.

- Conocer las demandas y las necesidades de los/ as jóvenes mayores de edad no emancipados de 18 a 25 años de la CAPV.

- Analizar la información y las demandas detectadas, recogiendo con carácter técnico las necesidades del colectivo juvenil, así como el ajuste entre éstas y la respuesta por parte de la Administración.

- Proponer orientaciones para elaborar políticas públicas que pudieran optimizar la intervención sobre este colectivo, así como servir de base para actuaciones futuras que mejoraran su situación.

3 Entendemos que éste es un compromiso de los/as educadores/as sociales en particular y de la educación social en general -colegios y asociaciones profesionales, Universidad-, como elementos de protección y acompañamiento dentro del Estado del bienestar.

\subsection{Metodología}

El planteamiento metodológico del estudio ha sido participativo, con una recogida de las necesidades tanto entre los jóvenes como entre los/as profesionales que intervienen con ellos/as, así como entre un grupo de expertos/as en intervenciones sociales con jóvenes y en el diseño y desarrollo de planes, programas y políticas de protección y promoción dirigidos a ellos/as. La metodología se organizó en torno a tres ejes:

- El Comité Asesor, un órgano conformado por diferentes agentes expertos en educación social con jóvenes en exclusión: profesionales de la administración pública o asesores técnicos de programas dependientes de ésta, el tercer sector, el Consejo Vasco de la Juventud, el Colegio de Educadores/as Sociales del País Vasco, el SIIS Centro de Documentos y Estudios, y de la Universidad (en concreto, de centros que imparten el grado de Educación Social: Escuela Universitaria de Magisterio de Bilbao, Facultad de Filosofía y Ciencias de la Educación -ambas de la Universidad del País Vasco- y Universidad de Deusto). Su función era, por un lado, aportar una visión general de la juventud de 18 a 25 años no emancipada desde el contraste en una entrevista personal; y por otro, asesorar sobre el borrador del estudio, mediante la participación en un grupo de discusión. En el Comité participaron 36 profesionales, estructurados en tres grupos, uno por cada territorio histórico.

- Un grupo de profesionales que desarrollaban intervenciones directas con jóvenes (trabajadores/as sociales, profesores/as, educadores/as sociales, mediadores/as). Tenía una doble función: aportar conocimiento desde su experiencia profesional, y conformar grupos de discusión o presentar grupos naturales de jóvenes para el desarrollo de entrevistas. En el grupo de profesionales participaron 26 personas.

- Entrevistas, grupos de discusión y contacto presencial con grupos naturales de jóvenes de siete localidades de la CAPV, con el objetivo de obtener de primera mano información sobre cuáles eran las necesidades reales de los/as jóvenes no emancipados/as de 18 a 25 años. Para ello, se hicieron entrevistas individuales aleatorias en la calle; se convocaron grupos de discusión (formados a través de la Universidad, programas de intervención social, y centros educativos y profesionales que trabajaban directamente con el colectivo, quienes conformaron grupos de carácter heterogéneo y con diversos perfiles sociales mediante convocatorias de carácter formal / reuniones) y se entrevistó in situ a grupos naturales en la calle y locales o lonjas (con los que nos pusieron en contacto fundamentalmente educadores/as de calle que participaron en el Grupo de Profesionales). En estas entrevistas, grupos de discusión y grupos naturales participaron unos trescientos jóvenes de los tres territorios 


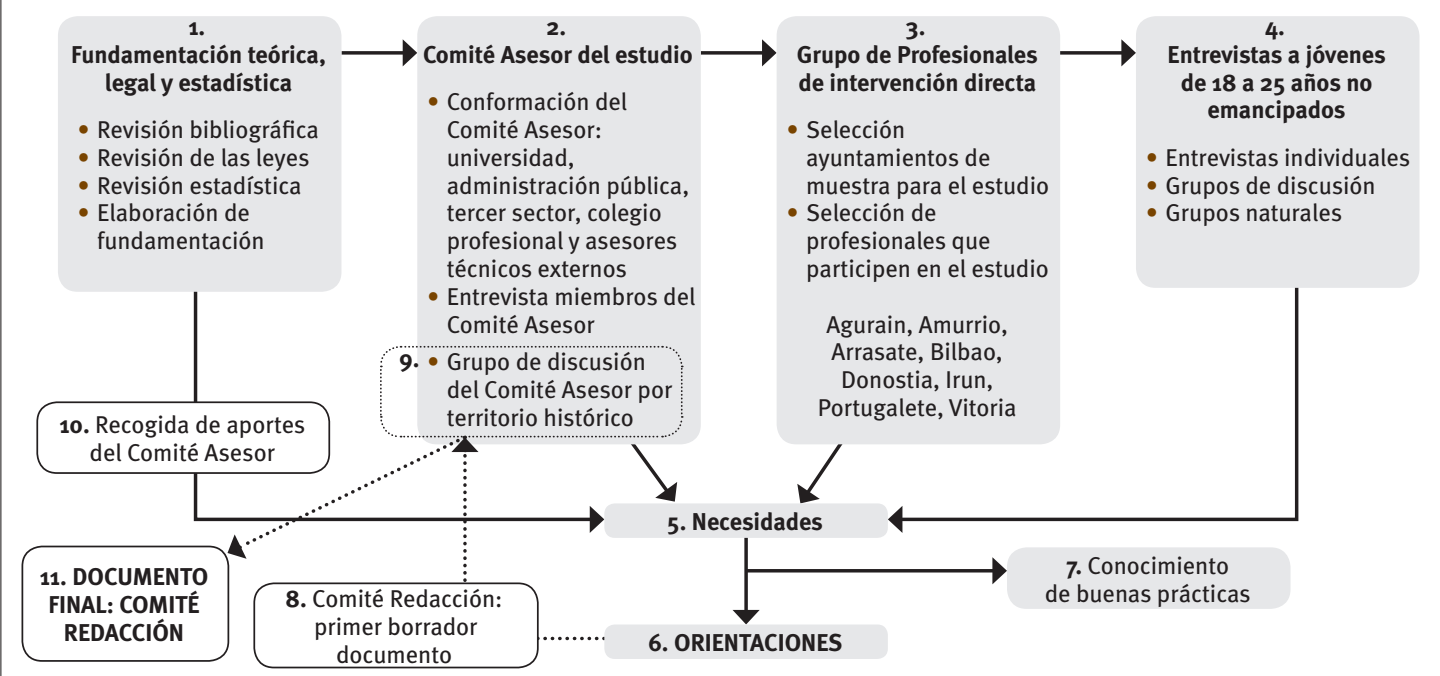

Fuente: Elaboración propia.

históricos. Las entrevistas se desarrollaron en siete municipios del País Vasco, elegidos según diferentes criterios, para garantizar la mayor representatividad posible de todos/as los/as jóvenes de la comunidad autónoma. Los criterios fueron los siguientes:

- Poblacionales: se escogieron algunas localidades con un alto número de habitantes.

- Geográficos: se procuró que no todos los municipios se concentraran en el Gran Bilbao, Donostialdea o Vitoria-Gasteiz.

- Nivel de exclusión: se recogió información en poblaciones, barrios o zonas con niveles de exclusión elevada, y en ayuntamientos con políticas proactivas con la juventud.

\subsection{Proceso de estudio}

El proceso de estudio aseguraba la recogida teórica de información, así como de muchos datos obtenidos del trabajo de campo desarrollado tanto con jóvenes como con profesionales. Para ello, se estructuró en tres fases bien definidas:

- En una primera fase, se llevó a cabo una fundamentación teórica a partir de bibliografía y fuentes documentales. Se tomaron como referencia las líneas estratégicas del III Plan de Inclusión Activa del Gobierno Vasco de cara al tratamiento de esa información, entendiendo que si el colectivo tenía dificultades de inclusión, se trataba de ver en torno a qué variables se medía la inclusión en el propio Plan, para verificar si estábamos en lo cierto, y si las respuestas al colectivo podían estar marcadas desde éste o era necesario incluir alguna nueva.

- En una segunda fase, se analizaron textos de carácter legal (leyes, órdenes, decretos, planes) que podían afectar al colectivo sujeto del estudio. Se plantearon los siguientes bloques temáticos: servicios sociales, educación, salud, drogodependencias, empleo, cultura y juventud.

\section{Figura 2. Fundamentación teórica}

Marco social y cultural en la transición de la vida adulta de los/as jóvenes de la CAPV

Relacionados con los objetivos planteados en el III Plan de Inclusión Activa de la CAPV

\section{Empleo}

Servicios sociales

Educación

Vivienda

Marco legal

Sanidad

Tercer sector

Planes
NECESIDADES

ORIENTACIONES

Fuente: Elaboración propia. 


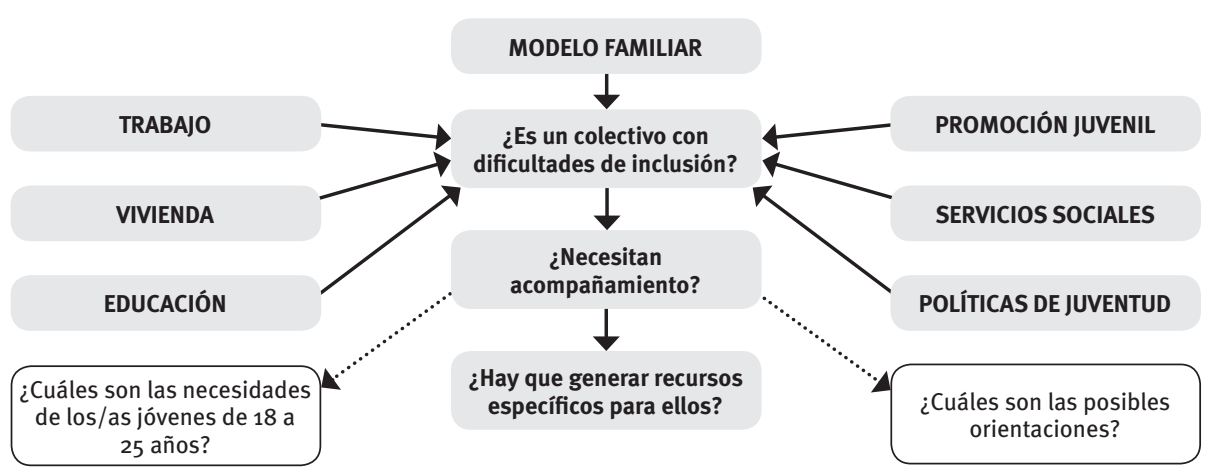

Fuente: Elaboración propia.

Por último, la tercera fase se centró en el trabajo de campo desarrollado mediante:

- Entrevistas personales a jóvenes, profesionales y miembros del Comité Asesor.

- Grupos de discusión de jóvenes.

- Entrevistas a grupos naturales in situ en el medio abierto: calle, locales o lonjas.

- Grupos de discusión del Comité Asesor, por territorio histórico, sobre el borrador de documento base generado con la información recogida.

En total, participaron más de 75 profesionales y unos/as 300 jóvenes. Se ordenó la información siguiendo el esquema de la Figura 3.

Este esquema sirvió como elemento de reflexión de los/as profesionales que conformaban el Comité Asesor, que concluyó que los/as jóvenes sí son un colectivo con dificultades de inclusión (al igual que otros), no tanto un grupo excluido; que el acompañamiento es muy necesario para aquellos/as que están en situación de dificultad; y que pueden existir recursos específicos para jóvenes, pero siempre orientados al acceso de la red de recursos y servicios normalizados, es decir, como transición hacia ésta. Debe buscarse que la red normalizada se ajuste a las necesidades de la población, y no tanto implementar recursos específicos para cada colectivo, como un elemento más para el tránsito de una comunidad excluyente a una comunidad inclusiva.

Los bloques en los que organizamos un primer borrador de documento para tratar en el Comité Asesor son una especie de cajones que ordenan las diferentes ideas en función de una serie de criterios que nos ayudaron a comprender con mayor precisión la realidad de los/as jóvenes y a extraer conclusiones que midieran su nivel de vulnerabilidad, la necesidad de intervenciones, qué aspectos habría que trabajar, o cuáles eran las carencias y déficits, entre otros aspectos. Con todo ello se pretendía agilizar la reflexión y ordenar las aportaciones de cada profesional, fomentando el debate en cada uno de los bloques y guiando el discurso hacia la extracción de orientaciones concretas para mejorar la atención a la población juvenil.

\subsection{Algunas conclusiones del estudio}

Mostramos aquí alguna de las conclusiones obtenidas en el estudio que definen la situación de dificultad en la inclusión de los/as jóvenes de la CAPV:

- Su situación individual está invisibilizada, ya que a efectos de 'números' o estadísticas, forman parte de la estructura familiar y no pueden nunca singularizarse dentro de ésta, lo que dificulta sus procesos de transición a la vida adulta. Esto da pie al desconocimiento de sus demandas y necesidades, ya que no hay instrumentos para canalizarlas y recogerlas, motivo por el que en ocasiones obtengan escasa respuesta por parte de la comunidad.

- Si bien es cierto que la crisis es un factor fundamental, pues se ha incrementado el número de jóvenes en situación de exclusión (sin recursos, sin trabajo, sin formación), no debemos olvidar que siempre ha habido situaciones de dificultad en el colectivo juvenil. Esta nueva realidad hace que se acentué la situación de exclusión de aquellos/as que ya lo estaban antes de la crisis, lo cual genera ‘una primera y segunda división' en situaciones de exclusión.

- A pesar de tener una situación 'cómoda' dentro del hogar familiar, sus niveles de autonomía son reducidos, lo cual crea expectativas y proyectos de vida poco definidos y con pocas expectativas de viabilidad.

- Las perspectivas de integración en el mercado laboral son exiguas (el $46 \%$ de la población de entre 18 y 25 años está en desempleo, sin contar a quienes están estudiando y no están inscritos en Lanbide) y el acceso a la vivienda es muy complicado (oferta de alquiler social reducida, elevado precio de la vivienda, falta de créditos bancarios). Por eso el inicio de procesos de vida autónomos 
resulta casi inviable, lo cual provoca frustración, apatía y abandono.

- Las respuestas por parte del sistema educativo no están ajustadas, siendo la única opción la educación reglada. Ello da lugar a dos situaciones: a) la creada por el alto nivel de fracaso de la escuela, de la que salen 'expulsados' un número importante de adolescentes y jóvenes, que quedan fuera del sistema formativo y -por falta de capacitación precisamente- también del laboral; y b) la de quienes permanecen dentro del sistema educativo de forma indefinida, esperando una oportunidad del mercado laboral (producen gasto en la familia y no ingresan nada).

- El nivel adquisitivo real de los/as jóvenes no emancipados es muy inferior al que que concebimos, ya que el dinero es fundamentalmente de las familias y apenas es gestionado por ellos/as mismos/as.

- Por otro lado, la falta de disponibilidad de tiempo por parte de los progenitores en etapas anteriores, como la infancia y la adolescencia, hace que en ocasiones no estén cubiertas las necesidades afectivo-emocionales.

- Asimismo, el número de recursos específicos donde los/as jóvenes pueden encontrar referentes cercanos, así como acceder a procesos de acompañamiento socioeducativos que los doten de información, orientación y seguimiento son muy limitados, al contrario que en otras etapas vitales (en la infancia, están los haurtxokos; en la adolescencia, los gaztelekus; y en la tercera edad, los hogar del jubilado).

- Los niveles de participación son muy bajos, y las estructuras participativas ajustadas a su perfil, deficitarias.

\subsection{Síntesis de las necesidades de los/as jóvenes de la CAPV obtenidas en el estudio}

A continuación exponemos una síntesis de las necesidades obtenidas del estudio (Ávila Ruiz, 2013):

- Una de las principales conclusiones es la imposibilidad de acceder a profesionales que los/as nutran de recursos, habilidades y herramientas para que desarrollen ciertas competencias, actitudes y aptitudes que favorezcan el aumento de su autoestima, madurez personal, motivación y empoderamiento. El refuerzo de estos elementos facilita el desarrollo de procesos de tránsito a la vida adulta normalizada, así como el establecimiento de itinerarios individualizados de carácter inclusivo.

- La necesidad de figuras de referencia en sus contextos cotidianos de relación -como la calle, los locales, los recursos o servicios- que acompañen a los/as jóvenes en la confección de proyectos de vida personalizados;

- La generación de modelos de intervención socioeducativos de carácter proactivo, que inciten a los/as jóvenes a construir, junto con los/as profesionales y la red de recursos y servicios normalizados, respuestas ajustadas a sus necesidades.

- Es imprescindible que la comunidad confíe en los procesos creados y desarrollados autónomamente por ellos/as mismos/as, que tienden a criminalizarse, como los locales o las actividades en la calle. Para legitimar la participación de los/as jóvenes en espacios comunitarios, es necesario confiar en ellos/as, que se sientan seguros, antes de responsabilizarlos/as de su no participación. La seguridad deriva en responsabilidad, la cual genera compromiso social y autonomía. Esto nos hace ver que, si reforzamos sus procesos autogestionados, se potenciará la comunidad y aumentará el índice de participación y aporte en el desarrollo de ésta.

- En el ámbito escolar, se aboga por revisar la formación general y específica, mientras que en el plano ocupacional se solicitan procesos de empleo-formación, así como impulsar las experiencias laborales.

- En cuanto a las políticas destinadas a los/as jóvenes de la CAPV, a pesar de que han tomado más cuerpo dentro de la Administración, sigue habiendo déficits: de vivienda, de empleo, de trasversalidad de las políticas juveniles, de análisis de la juventud actual -que necesita una reinterpretación y un reconocimiento nuevo-, de aplicación de un enfoque comunitario y un desarrollo sistémico, de intervenciones basadas en la relación educativa y, por último, de creación de procesos dentro de la propia comunidad y en la vida cotidiana. Asimismo, se transmite la necesidad de dar un mayor protagonismo al mundo juvenil en la elaboración de políticas y planes que lo atañen, lo cual requiere su participación en las instituciones, tanto en el plano técnico como político.

- Reforzar las políticas de servicios sociales y promoción juvenil a efectos tanto de cobertura de situaciones de riesgo como de participación de los/as jóvenes.

- Ajustar los recursos y servicios dirigidos a la población juvenil. A este respecto, se recomienda, entre otras cuestiones: el análisis y diagnóstico previo de la realidad a la hora de poner en marcha servicios; que las intervenciones no sean tan sólo de carácter informativo, sino que se compongan de un servicio de acompañamiento socioeducativo en que la información sea una acción más del proceso; un mayor número de canales de interlocución.

- Necesidad de integrar la perspectiva de género y la interculturalidad.

\subsection{Síntesis de las orientaciones del estudio}

Presentamos aquí una síntesis de las orientaciones:

- Fomentar programas que posibiliten procesos de participación y promoción de los/as jóvenes. 
- Adaptar o crear recursos específicos para la inserción laboral y la inclusión social, ajustar los recursos de formación y empleo, y optimizar las políticas de vivienda (entendiendo que los elementos estructurales son básicos para generar procesos inclusivos).

- Fortalecer las políticas de intervención con continuidad en las fases anteriores a la adultez.

- Abrir canales de comunicación para la conformación de la oferta.

- Redefinir las ayudas económicas.

- Adaptar los recursos de información como recursos de acompañamiento (la información tan sólo es una acción más del acompañamiento socioeducativo).

- Desarrollar intervenciones específicas con jóvenes en exclusión social: implantar programas de intervención socioeducativa en medio abierto con jóvenes y reforzar los programas de intervención socioeducativa con familias con jóvenes a cargo en situación de riesgo.

\section{Fase de intervención: programa piloto de educación de calle de Debabarrena}

\subsection{Fundamentación}

Recogiendo la última de las orientaciones del estudio, desde la entidad se planteó el desarrollo de un programa de intervención socioeducativa en medio abierto con jóvenes en exclusión de 18 a 30 años en la comarca de Debabarrena. Para ello, se puso en marcha un programa piloto con la financiación del Área de Inclusión de la Diputación Foral de Gipuzkoa y del Departamento de Servicios Sociales del Ayuntamiento de Elgoibar, mediante la contratación de dos educadores sociales a tres cuartos de jornada.

El programa buscaba el ajuste de la respuesta a las necesidades los/as jóvenes en exclusión y se caracterizaba por:

- Desarrollar las intervenciones en el medio abierto, especialmente en espacios como la calle o lugares de reunión de los/as jóvenes. El educador se erigía en un referente cercano y disponible para los/as jóvenes, en contacto real con la comunidad y que acudía a los sitios donde se desarrollaba la vida cotidiana de las personas (al contrario que muchos servicios que, para utilizarse, requieren acudir a sus espacios).

- Basarse en la relación educativa como principal herramienta de intervención. Desde esta perspectiva, se entiende que la intervención con los/as jóvenes deriva del compromiso fundado en una relación que, primero, convierte al profesional en referente, y luego, en una persona ascendente que posibilita el desarrollo de procesos de acompañamiento de carácter integral.
- Llevar a cabo las intervenciones en un proceso ajustado a las necesidades y capacidades de cada uno/a de los/as jóvenes atendidos, siempre desde un modelo proactivo y en coordinación con el resto de agentes, recursos y servicios corresponsables de los/as jóvenes.

- Perseguir el cambio y, por lo tanto, adoptar un enfoque técnico basado en una planificación y un diseño de proyectos educativos que posibiliten el desarrollo de intervenciones de calidad.

- Basarse en un modelo de capacitación, es decir, volcado en el abono de las capacidades de los/as propios jóvenes para compensar las dificultades 0 necesidades existentes.

La comarca donde se desarrolló el servicio, Debabarrena, se encuentra en el extremo noroeste de la provincia de Gipuzkoa y cuenta con unos 60.000 habitantes. Está conformada por los municipios de Deba, Eibar, Elgoibar, Mendaro, Mutriku y Soraluze, y tiene un carácter predominantemente industrial. La duración del programa piloto se estipuló en seis meses, tiempo que permitiría iniciar procesos inclusivos con jóvenes. El programa se ubicaba en los servicios sociales de Elgoibar, aunque atendía a jóvenes de toda la comarca. El proyecto piloto ha derivado en la creación de un servicio de acompañamiento especializado a jóvenes, totalmente ajustado a las necesidades de éstos/as, para el establecimiento de itinerarios inclusivos.

Los destinatarios de la intervención son los/as jóvenes de 18 a 30 años preferentemente, así como los recursos y servicios corresponsables de esta población de la comarca de Debabarrena (y, por extensión, toda la comunidad). Algunos de los indicadores más habituales en los/as jóvenes con los que se trabaja son: inactividad, ausencia de ocupación laboral o formativa; causas judiciales pendientes; bajos índices de motivación y, como consecuencia, autoconcepto inadecuado; residencia itinerante y falta de ingresos; problemas en la documentación (sin empadronamiento, DNI, ni tarjetas de Lanbide o Osakidetza); estigmatización por parte de la comunidad.

\subsection{Objetivos}

Este programa piloto se ha planificado en torno a tres líneas estratégicas de intervención, que se han marcado tras un pequeño estudio de la comarca para adaptar las conclusiones obtenidas en el estudio general a las especificidades de los/as jóvenes de esta zona. Dichas líneas estratégicas son:

1. Dar respuesta a las necesidades socioeducativas de los/as jóvenes con dificultades de inclusión, previa identificación de aquéllas en sus espacios cotidianos de relación, el desarrollo de procesos educativos individuales y la puesta en marcha de marcos grupales, que han abordado diferentes cuestiones (inserción sociolaboral, motivación, habilidades sociales, conocimiento de recursos, elaboración de itinerarios personales). 
2. Fomentar la adaptación de los recursos y la creación de otros nuevos para el desarrollo de proyectos educativos de calidad que faciliten la futura participación de los/as jóvenes en espacios normalizados (coordinación con recursos, servicios y empresas de inserción sociolaboral).

3. Crear una estructura de coordinación con las diferentes administraciones responsables de las respuestas a los/as jóvenes (ayuntamientos, diputaciones, Gobierno Vasco-Lanbide).

\subsection{Metodología}

La planificación diseñada por el equipo educativo se ha llevado a cabo de acuerdo a una metodología organizada en bloques de acción, con contenidos de trabajo y temporalización ajustados:

- Conocimiento de la zona y adaptación del estudio a las necesidades de la población concreta.

- Detección de los/as jóvenes en situación de exclusión en sus espacios cotidianos de actividad, principalmente la calle. También se ha atendido a aquellos/as jóvenes derivados por los servicios sociales de base (aunque la mayoría no acuden a ellos, ya que son sus padres o madres quienes lo hacen).

- Establecimiento de la relación educativa con los/ as jóvenes en sus espacios cotidianos de desarrollo: conocimiento, presentación de la figura del educador, delimitación de roles, normas de relación, compartir tiempo.

- Diagnóstico de necesidades: se ha utilizado el Instrumento de Valoración de las Situaciones de Exclusión Social de Gobierno Vasco (Decreto 353/2013), acompañado de unos indicadores generados por la entidad que facilitan el diseño del proceso individual. Este diagnóstico se ha realizado generalmente de manera compartida con el trabajador o trabajadora social de referencia de los servicios sociales de base.

- Diseño y desarrollo de los planes individuales de atención (PIA): cada joven con quien se ha intervenido ha tenido un plan individualizado, que contemplaba tanto indicadores objetivos como elementos cualitativos definidos en las diferentes áreas de intervención, en que se desarrolla su vida (individual, social, familiar, formativolaboral, judicial, vivienda, tiempo libre). En este plan se marcaban tanto objetivos como acciones a desarrollar y tiempos. Se consensuaba con el/la propio/a joven, y recogía compromisos por su parte y por parte del equipo educativo.

- Creación de marcos socioeducativos grupales: se han creado grupos para el desarrollo de los planes de atención individual, como elemento de 'ensayo previo' a su inclusión en recursos normalizados. En ellos no sólo han participado jóvenes en exclusión, sino también otros/as con situaciones normalizadas, por entender que el propio marco grupal es un elemento de retroali- mentación educativa que enriquece a todos/as los/as participantes.

- Coordinación con los servicios sociales de base y los servicios sociales especializados: se han realizado reuniones de coordinación con los/as trabajadores/as sociales de referencia de los/ as jóvenes, que son, en la gran mayoría de los casos, los/as profesionales que los/as acercan a los servicios sociales. La intervención en cada uno de los PIA se ha intentado enmarcar dentro de los convenios de inserción de los/as trabajadores/as sociales, con el fin de desarrollar intervenciones globalizadas e integrales que potencien los recursos de acompañamiento.

- Trabajo comunitario: se han desarrollado acciones de coordinación con todos los agentes, recursos, servicios y departamentos responsables de los/as jóvenes (abogados, fiscalía, recursos de empleo, formativos, policía municipal, agencias de desarrollo comarcal), que han actuado como eje para el desarrollo de intervenciones integrales desde todos aquellos espacios donde se desarrolla el/la joven. Asimismo, se han generado procesos de adaptación de los propios recursos y servicios a las necesidades de los/as jóvenes, para un desarrollo más óptimo de éstos/as. Por último, se ha derivado a jóvenes hacia recursos normalizados, intentando desarrollar procesos transitorios de acompañamiento por parte del equipo educativo.

\subsection{Algunas acciones}

Entre otras, se han realizado las siguientes intervenciones:

- Acompañamiento y orientación individual a los/as jóvenes en diferentes contextos: dificultades familiares, procesos judiciales, recursos formativos, charlas y reflexiones.

- Procesos grupales de socialización: se pretende crear espacios con grupos naturales o construidos por el equipo educativo donde abordar conflictos, preocupaciones, dificultades.

- Formación: preparación de exámenes para acceso a niveles de cualificación dentro del sistema de formación ofertado por Lanbide-Sistema Vasco de Empleo.

- Actividades de tiempo libre saludables: realización de actividades de ocio que fomenten el contacto con la naturaleza, la cultura, así como la reducción de consumos.

- Rehabilitación del local: habilitación de un local cedido por el Ayuntamiento de Elgoibar para crear un punto de encuentro, un lugar para dinámicas grupales o realización de talleres.

- Reutilización de residuos: desarrollo de talleres de carácter ecológico y formativo para la reutilización de residuos (construcción de muebles con palés, 'tuneado' de bicis, elaboración de jabón). 
- Medidas judiciales: cumplimiento de medidas judiciales alternativas al internamiento con algunos/as jóvenes de la comarca.

\section{Fase de evaluación: orientaciones a futuro}

El equipo educativo se encuentra actualmente en la fase de evaluación del programa piloto, con el afán de poder estabilizarlo como servicio de acompañamiento especializado. La respuesta dada por el programa a los objetivos planteados ha sido muy satisfactoria, así como la acogida por parte de los/as jóvenes de la comarca de un servicio de acompañamiento socioeducativo de este tipo. Aun así, debemos hacer un contraste más pausado del proceso de evaluación, pero sí nos suscita una nueva reflexión que queremos trasladar aquí, una nueva oportunidad de investigar para generar elementos de mejora aplicables en la siguiente intervención.

Si bien es cierto que el acompañamiento es muy satisfactorio para el desarrollo de procesos inclusivos con jóvenes, también vemos necesario reforzar elementos estructurales que puedan potenciar ese paso, es decir, generar procesos de inserción laboral que permitan su acceso al mercado laboral y posibiliten experiencias de emancipación ordenadas y con supervisión. Estos elementos estructurales (trabajo/formación y vivienda), junto con procesos educativos de carácter individual, social y de empoderamiento, posibilitarían la integración comunitaria de éstos jóvenes en exclusión. Por ello, planteamos el esquema de la Figura 4, que es el elemento de partida para elaborar un programa de intervención socioeducativa para la inclusión con plenas garantías de éxito.
La nueva reflexión viene dada por que un acompañamiento sin recursos de carácter estructural con jóvenes, en ocasiones, no es suficiente para abordar intervenciones integrales que garanticen tránsitos de calidad a la vida adulta. Por ello, nos planteamos, como nueva reflexión, llevar a cabo intervenciones socioeducativas en espacios de carácter más estructural, como la formación/trabajo o la vivienda. Evidentemente, esta reflexión está anclada en la comarca en la que desarrollamos el proyecto, porque en otras comarcas o territorios históricos ya están en marcha servicios como el que planteamos.

En esta primera reflexión para delimitar un proceso de investigación, ordenamos las ideas desde el esquema planteado anteriormente, en el que sería necesario un modelo en el que el centro de la intervención sería la persona, que podría disfrutar de acompañamientos en diferentes espacios, como el más personal/social, el laboral y el de la vivienda.

Cada uno de estos espacios nos plantea un nuevo objetivo a la hora de desarrollar una reflexión enfocada a la acción, que estructuramos de la siguiente manera:

- Factores humanos: en este primer espacio, se aboga por continuar con el desarrollo de proyectos de intervención socioeducativa en el medio abierto, entendiendo éste como aquellos contextos donde se desarrolla la vida de las personas (donde adquieren herramientas, habilidades y recursos para su desarrollo). Como decíamos antes, la experiencia totalmente satisfactoria de la puesta en marcha del proyecto piloto nos hace abogar por la potenciación de estos proyectos, acompañándolos, quizás en ocasiones, de procesos de mediación o intervención familiar, para trabajar aspectos dentro de este contexto concreto.

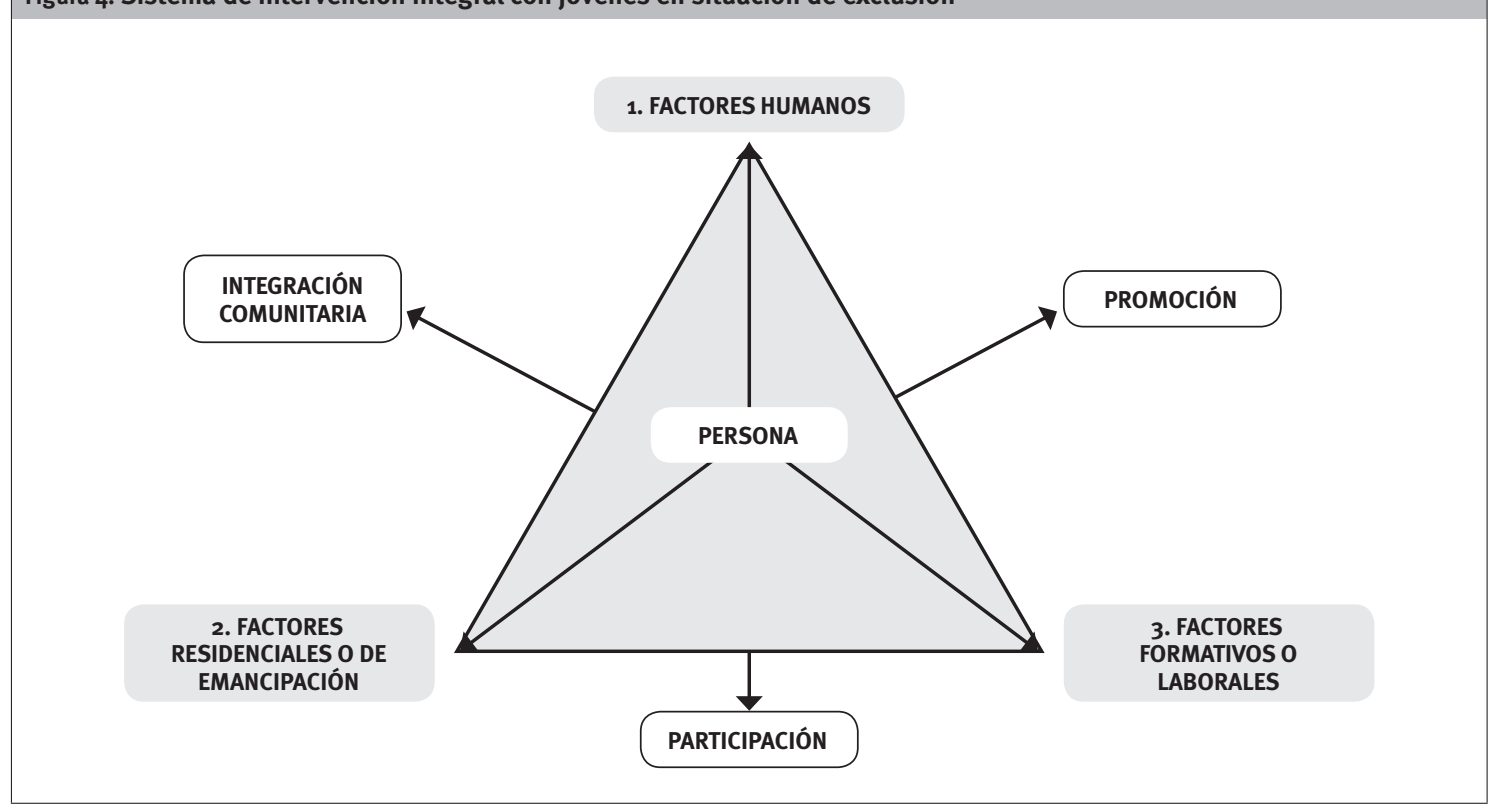

Fuente: Elaboración propia. 
- Factores residenciales o de emancipación: la imposibilidad de muchos/as jóvenes de desarrollar proyectos de emancipación por motivos económicos (falta de acceso al mercado laboral y al sistema de prestaciones), motivos de madurez personal y, sobre todo, por factores organizativos (gestión de recursos, convivencia) hace necesario el establecimiento de proyectos de intervención en pisos, hogares o recursos residenciales con personas en situación de exclusión. Éstos serían tan sólo un espacio previo y de transición a la vida autónoma, en el que se abordarían diferentes aspectos y donde se transferirían actitudes y aptitudes para potenciar procesos autónomos de transición a la vida adulta.

- Factores laborales o formativos: finalmente, hemos observado la importancia de desarrollar procesos formativos o laborales específicos para jóvenes con dificultades de inclusión. Estos procesos garantizan dos cosas: por un lado, la posibilidad de acceder a dinero para ser autónomos e iniciar una emancipación; y por otro, algo básico, la ubicación del mundo de trabajo dentro de la organización del / de la joven, al situar el trabajo como elemento de empoderamiento y participación social, así como de reconocimiento y satisfacción social (no tan sólo como una forma de conseguir dinero). Por ello, vemos necesario iniciar otra reflexión en torno al desarrollo de proyectos de intervención socioeducativa en contextos laborales o de capacitación/formación.

Entendemos que la intervención en estos tres espacios para trabajar factores de diverso tipo debe estar integrada en un proyecto que cubra las necesidades en todos los contextos, de forma que esta intervención macro produzca resultados como la participación, la promoción y la integración comunitaria. Éste es, en la actualidad, uno de los nuevos debates y retos a abordar en nuestros espacios de reflexión, que, a través de un proceso de definición técnico, nos abocará al desarrollo de nuevas investigaciones enfocadas a poner en marcha experiencias que siempre reviertan en la mejora de la calidad de vida de los/as ciudadanos/as.

\section{Bibliografía}

(2013): "Decreto 353/2013, de 28 de mayo, de Ficha Social del Sistema Vasco de Servicios Sociales y del instrumento de diagnóstico social del Sistema Vasco de Servicios Sociales", Boletín Oficial del País Vasco, no 121, 26-6-2013 [<http://www. lehendakaritza.ejgv.euskadi.net/r48-vacia/es/ bopv2/datos/2013/06/1302907a.pdf〉].

(2008): “Ley 18/2008, de 23 de diciembre, para la Garantía de Ingresos y para la Inclusión Social", Boletín Oficial del País Vasco, nํ250, 31-122008, págs. 32.729-32.804 [‘http://www. lehendakaritza.ejgv.euskadi.net/r48-vacia/es/ bopv2/datos/2008/12/0807235a.pdf)].

(2008): "Ley 12/2008, de 5 de diciembre, de Servicios Sociales", Boletín Oficial del País Vasco, n- 246, 24-12-2008, págs. 31.840-31.924 [<http://www.euskadi.net/bopv2/ datos/2008/12/0807143a.pdf>].

ÁVILA RUIZ, A. (coord.) (2013): Estudio de necesidades de jóvenes mayores de edad de 18 a 25 años no emancipados de la CAPV, Urnieta, Kalexka Elkartea [khttp://www.kalexka.com/upload/ articulos/Estudio_de_necesidades_de jovenes_mayores_de_edad_de_18_a_25_a_ os_no_emancipados_de_la_CAPV.pdf $\rangle$ ].

CONSORCIO PARA LA EDUCACIÓN COMPENSATORIA Y LA FORMACIÓN OCUPACIONAL DE GIPUZKOA (2001): Programa Marco de Intervención
Socioeducativa, desde los Servicios Sociales Comunitarios, con Menores y Jóvenes en Situación de Riesgo.

GOBIERNO VASCO (2012): III Plan de Inclusión Activa 201216 de la CAPV, Vitoria-Gasteiz, Servicio Central de Publicaciones del Gobierno Vasco.

KALEXKA ELKARTEA (2009): Estudio de necesidades de niños-as y adolescentes en situación de riesgo del municipio de Elgoibar, Ayuntamiento de Elgoibar.

MORENO MÍNGUEZ, A. (2012): La transición de los jóvenes a la vida adulta, Barcelona, Obra Social La Caixa.

OTAÑO, J. (2012): “Nuevas formas de exclusión social como consecuencia del fuerte incremento del desempleo. Comparecencia en el Senado, ante la Comisión Especial sobre las nuevas formas de exclusión social consecuencia del fuerte incremento del desempleo, el 12 abril 2010, en representación del Consejo General de Educadoras y Educadores Sociales", Revista de Educación Social, no 14 [rhttp://www.eduso. net/res/pdf/14/nuefor_res_14.pdf $>$ ].

- (2006): Una reflexión sobre las claves de la intervención socioeducativa a través de sus tres herramientas de trabajo. Educación de calle en Andoain, 1994-2006, Ayuntamiento de Andoain; Diputación Foral de Gipuzkoa. 